\title{
Charge Transport and Electrochromism in Novel Nanocomposite Films of Poly(3,4-ethylenedioxythiophene)-Au Nanoparticles-CdSe Quantum Dots
}

\author{
Shweta Bhandari, ${ }^{\dagger}$ Melepurath Deepa, ${ }^{*, *}$ Shailesh N. Sharma, ${ }^{\dagger}$ Amish G. Joshi, ${ }^{\dagger}$ \\ Avanish Kumar Srivastava, ${ }^{\dagger}$ and Rama Kant ${ }^{\S}$ \\ National Physical Laboratory, Dr. K.S. Krishnan road, New Delhi-110012 India, Department of Chemistry, \\ Indian Institute of Technology Hyderabad, Ordnance Factory Estate, \\ Yeddumailaram-502205, Andhra Pradesh, India, and Department of Chemistry, University of Delhi, \\ New Delhi-110007, India
}

\begin{abstract}
Nanocomposite films of Poly(3,4-ethylenedioxythiophene)-Au-CdSe have been fabricated for the first time by first growing a porous PEDOT layer in the ionic liquid 1-butyl-1-methylpyrrolidinium bis(trifluoromethylsulfonyl)imide and then introducing the film to a mixed colloid of Au nanoparticles (NPs) and CdSe quantum dots (QDs), thereby imparting a uniform confinement of Au NPs/CdSe QDs nanocomposite to the bulk of the polymer film by the virtue of affinity that $\mathrm{Au}$ has for the sulfur on the thiophene rings. Progressive luminescence quenching and absorption enhancement of CdSe QDs as a function of Au NPs incorporation in the CdSe colloid confirmed the direction of charge transfer from CdSe QDs to Au NPs. The optimized composition of $\mathrm{Au}-\mathrm{CdSe}$ based on a maximum charge transfer was utilized for the formation of the nanocomposite with PEDOT. The loss of electrons from CdSe in the nanocomposite was also reflected in the core level signals of Se $3 \mathrm{~d}$ corresponding to oxidized states of $\mathrm{Se}^{2+}$ and $\mathrm{Se}^{0}$. The charge propagation from $\mathrm{CdSe}$ to $\mathrm{Au}$ to PEDOT through Fermi-level equilibration was also confirmed by luminescence and absorption spectroscopy. The effect of $\mathrm{Au}-\mathrm{CdSe}$ on electrochromic performance of the nanocomposite film was realized in terms of a superior optical density change, larger coloring efficiency $\left(300 \mathrm{~cm}^{2} \mathrm{C}^{-1}, \lambda=550 \mathrm{~nm}\right)$, and faster colorbleach kinetics when compared with the electroactivity of the control PEDOT film, grown without the inclusion of any nanomoiety.
\end{abstract}

\section{Introduction}

In the past decade, ${ }^{1-3}$ nanomaterials have aroused significant research interest because of their unique size-dependent electronic, magnetic, optical, and electrochemical properties. Combination of different materials on the nanoscale has also attracted immense attention because new properties emerge in the resulting nanocomposites. To this end, nanocomposites of conducting polymers and metal nanoparticles $(\mathrm{NPs})^{4-6}$ or semiconducting quantum dots (QDs) ${ }^{7}$ have been extensively studied in the past. Tuning the properties of these nanocomposites by changing shape or size of the NPs and different combinations of the two constituents holds the key to unlock incredible possibilities in the field of science and technology.

Noble-metal NPs are known to be chromophores in the visible and near-infrared regions because of the excitation of localized surface plasmons. ${ }^{8}$ When the plasmon absorption of a metal $\mathrm{NP}$ and the absorption maximum of a doped conducting polymer match, the NP acts to enhance the absorption maximum of the polymer which can effectively enhance the coloration density, while evaluating the electrochromic response of the polymer by applying bias. Au NPs with diameters in the range of 5-50 nm have been reported to show $\lambda_{\max }$ between 520 and $530 \mathrm{~nm},{ }^{9}$ but such a chromophoric effect can only be observed when the incident wavelength is greater than the diameter of the NP. ${ }^{10}$
The use of these metal NPs in conjunction with polymers for chromic applications has already been reported in literature in the past. ${ }^{11}$ Recently, Leroux et al. ${ }^{12}$ demonstrated an active plasmonic device based on a Au-NP grating by using a conducting polymer (electrochemical switching). Besides this, metal NPs have also been reported to show an increased electronic conductivity in composites via increased coupling afforded by conjugated polymers. ${ }^{13}$ Sarma $^{14}$ and Zhai et al. ${ }^{15}$ observed a two-order increment in conductivity, when Au NPs were incorporated in polyaniline and polythiophene. In a manner akin to these metallic NPs, semiconducting QDs also act as efficient conduits for fast-electron carrier transport. Because of their size-dependent color tunability, large photostability, and quantum efficiency, they find use in a variety of applications including luminescent devices, biological markers, lasers, and catalysis. ${ }^{16-19}$ Sofos et al. $^{20}$ were successful in synthesizing lamellar conjugated molecule/ZnO hybrid materials that showed a stable photoconductive performance. In another study, Wang et $\mathrm{al}^{7}$ fabricated multilayer films of CdSe QDs and poly(3,3( 3 '-thienyloxy)propyltrimethylammoniumbromide) capable of a very high incident-photon-to-current conversion efficiency, which was over $6 \%$.

If a typical characteristic of a noble metal NP, namely, surface plasmon resonance, is integrated with that of semiconductor QDs, that is, luminescence, an exciting possibility can be realized. Previously, composites of conducting polymer either with $\mathrm{Au}^{21,22}$ or $\mathrm{CdSe}^{23,24}$ or $\mathrm{Au}--\mathrm{CdSe}$ without polymer ${ }^{25,26}$ have been fabricated, but a system involving a conducting polymer combined with $\mathrm{Au}-\mathrm{CdSe}$ has not been attempted so far. In the present report, we have used metallic Au NPs and semiconduct- 
ing CdSe QDs to form composites with electrochromic conducting polymer $\{$ Poly-3-4-ethylenedioxythiophene (PEDOT) $\}$. The charge-transfer phenomena between the various constituents of the ensuing nanocomposite have been followed by luminescence quenching and X-ray photoelectron spectroscopy. The composite shows an enhanced conductivity and an amplified electrochromic contrast leading to a high coloring efficiency.

\section{Experimental Section}

2.1. Materials. 3,4-Ethylenedioxythiophene or EDOT, cadmium oxide (CdO), trioctyl phosphine oxide (TOPO), trioctyl phosphine (TOP), selenium powder, and hydrogen tetrachloroaurate $\left(\mathrm{HAuCl}_{4}\right)$ were purchased from Aldrich. The ionic liquid 1-butyl-1-methylpyrrolidinium bis(trifluoromethylsulfonyl)imide, tetraoctylammonium bromide, sodium borohydride $\left(\mathrm{NaBH}_{4}\right)$, and anhydrous sodium sulfate $\left(\mathrm{Na}_{2} \mathrm{SO}_{4}\right)$ were obtained from Merck. Tetradecylphosphonic acid was procured from Alfa Aesar. All chemicals were used as received. Inorganic transparent electrodes of $\mathrm{SnO}_{2}: \mathrm{F}$ coated glass (Pilkington, sheet resistance: $14 \Omega / \mathrm{sq}$ ) were cleaned in a soap solution, $30 \% \mathrm{HCl}$ solution, double-distilled water, acetone, and trichloroethylene (in that order) prior to use. Deionized water (resistivity: $\sim 18.2$ $\mathrm{M} \Omega \mathrm{cm}$ ) obtained through Milli-Q system, methanol (Merck), dimethyformamide (DMF, Merck), and toluene (Spectrochem) were used as solvents.

2.2. Preparation of CdSe QDs. CdSe QDs were synthesized by a chemical route using a TOP/TOPO capping method reported previously. ${ }^{27} \mathrm{CdO}(0.02 \mathrm{~g})$, tetradecylphosphonic acid $(0.11 \mathrm{~g})$, and TOPO $(1.88 \mathrm{~g})$ were introduced into a $250 \mathrm{~mL}$ flask and heated to $\sim 320{ }^{\circ} \mathrm{C}$ under inert atmosphere with continuous stirring. After the dissolution of $\mathrm{CdO}$, the solution was cooled to $270{ }^{\circ} \mathrm{C}$, and a solution of Se powder $(0.02 \mathrm{~g})$ in TOP $(1.2 \mathrm{~mL})$ was loaded into the flask. The solution was heated at $250{ }^{\circ} \mathrm{C}$ for $5 \mathrm{~min}$ and then cooled to room temperature. As,prepared CdSe QDs were washed with methanol to remove excess TOPO. The final CdSe QDs were dispersed in toluene for further use.

2.3. Preparation of Au NP Dispersion. Stable solutions of $\mathrm{Au}$ NPs in toluene were prepared by using a method reported by Brust et al. ${ }^{28}$ Solutions of $\mathrm{HAuCl}_{4}(0.18 \mathrm{~g})$ in $15 \mathrm{~mL}$ of deionized water and tetraoctylammonium bromide $(1.09 \mathrm{~g})$ in $40 \mathrm{~mL}$ of toluene were mixed and stirred vigorously for about 10 min until the color of the aqueous phase became clear and that of the organic phase became orange, ensuring the complete transfer of $\mathrm{AuCl}_{4}^{-}$into the organic phase. Thereafter, a solution of $\mathrm{NaBH}_{4}(0.4 \mathrm{M})$ in deionized water was added slowly to the toluene phase (preferably dropwise) with continuous stirring. The color of the solution changed from orange to ruby red whereas the lower aqueous phase turned colorless. The mixture was stirred for 30 more minutes before the final separation of the two phases. The organic phase containing Au NPs was extracted, washed once with dilute sulfuric acid for neutralization and subsequently five times with distilled water, and then dried with anhydrous sodium sulfate. The resulting ruby-red dispersions were stored in dark at room temperature.

2.4. Fabrication of PEDOT-CdSe-Au Nanocomposites. A solution of $0.1 \mathrm{M}$ EDOT in 3.3 M 1-butyl-1-methylpyrrolidinium bis(trifluoromethylsulfonyl)imide and DMF ( $4: 1$ by volume) was prepared for the deposition of PEDOT films. The solution was magnetically stirred at room temperature for 20 min before deposition. The films of PEDOT were fabricated at room temperature $\left(25 \pm 2{ }^{\circ} \mathrm{C}\right)$ in a rectangular glass cell with a transparent conducting substrate $\left(\mathrm{SnO}_{2}: \mathrm{F}\right.$ coated glass or FTO $)$ as the cathode, a Pt sheet as the counter electrode, and a $\mathrm{Ag}$ / $\mathrm{AgCl} / \mathrm{KCl}$ reference electrode under potentiostatic conditions
$(+1.2 \mathrm{~V})$ for about $8 \mathrm{~min}$. The total volume of the polymerization solution was $50 \mathrm{~mL}$. The size of the FTO plate was 4 $\mathrm{cm} \times 3 \mathrm{~cm}$, the substrate thickness was about $4 \mathrm{~mm}$, and the thickness of the FTO coating was about $1 \mu \mathrm{m}$. The resulting blue-colored films of PEDOT were immediately rinsed with deionized water and ethanol and dried in air for two hours and stored in air. The neat PEDOT films are referred to as PEDOT (IL), and the average thickness was found to lie in the range of $600-650 \mathrm{~nm}$, as determined by talystep.

To make nanocomposites, dispersions of Au NPs and CdSe QDs were mixed together (1:1 by volume), and the resulting solution was drop-cast on PEDOT (IL) films and left undisturbed for $24 \mathrm{~h}$ in dark. Subsequently, the films were washed with deionized water and dried in air prior to use. The PEDOT (IL)$\mathrm{Au} \mathrm{NPs} / \mathrm{CdSe}$ QDs films are hereafter referred to as PEDOT(IL)- $\mathrm{Au}-\mathrm{CdSe}$ nanocomposite. Similarly, PEDOT (IL)$\mathrm{Au}$ and PEDOT (IL)-CdSe films were also fabricated by the solution casting method, from their respective suspensions.

2.5. Characterization Techniques. Photoluminescence (PL) measurements were carried out on a Perkin-Elmer LS-55 Spectrometer at room temperature. For transmission electron microscopy (TEM), thin layers of the samples were carefully extracted in deionized water and then transferred onto carboncoated copper grids, $3.05 \mathrm{~mm}$ in diameter, and the solvent was evaporated before use. TEM was carried out on a transmission electron microscope HRTEM Tecnai $\mathrm{G}^{2}$ F30 STWIN with a FEG source at $300 \mathrm{kV}$. XPS spectra were recorded for the assynthesized PEDOT films by using a Perkin-Elmer 1257 model operating at a base pressure of $7.8 \times 10^{-8}$ Torr at $300 \mathrm{~K}$ with a nonmonochromatized $\mathrm{AlK}_{\alpha}$ line at $1486.6 \mathrm{eV}$, an analyzer pass energy of $60 \mathrm{eV}$, and a hemispherical sector analyzer capable of $25 \mathrm{meV}$ resolution. The overall instrumental resolution was about $0.3 \mathrm{eV}$. The core-level spectra were deconvoluted by using a nonlinear iterative least-squares Gaussian fitting procedure. For all fitting doublets, the FWHMs were fixed accordingly. In order to confirm that the $\mathrm{Au}-\mathrm{CdSe}$ nanostructures are distributed across the cross-section of the film, the composite film was also subjected to sputtering with an argon-ion beam of $4 \mathrm{keV}$ energy and $20 \mathrm{~mA}$ emission current from a differential pumped ion gun (model: 04-300) operating in raster mode at a base pressure maintained at $1.8 \times 10^{-6}$ Torr. A $10 \mathrm{~nm}$ etching was achieved after the sputtering, and XPS spectra of the composite were recorded after the sputtering. Absorption spectra of solutions and optical density of films as a function of applied potential was measured ex-situ on a PerkinElmer Lambda 25 spectrophotometer in $3.3 \mathrm{M}$ 1-butyl-1methylpyrrolidinium bis(trifluoromethylsulfonyl)imide. An automated set up comprising a $\mathrm{He}-\mathrm{Ne}$ laser $(\lambda=632.8 \mathrm{~nm})$, a Si photodetector, and a custom-made microprocessor controlled versatile unit was used to record the switching time characteristics for the films between colored and bleached states.

\section{Results and Discussion}

3.1. PL and absorbance studies of $\mathbf{A u}-\mathrm{CdSe}$ colloids. In order to optimize the relative composition of $\mathrm{Au}$ and $\mathrm{CdSe}$ prior to the incorporation in PEDOT, control experiments were carried out. Luminescence quenching of CdSe as a function of increasing Au concentration to the CdSe QD dispersion of a given concentration is shown in Figure 1a. The CdSe QD solution strength was $20 \mathrm{mM}$ in toluene, to which a $12.9 \mathrm{mM}$ AuNP solution was added progressively; the total volume of the solution was kept constant. The neat $\mathrm{CdSe}$ solution shows a sharp emission peak at $\lambda_{\max }=521 \mathrm{~nm}$, and upon introducing Au NPs, the peak intensity decreases. It is well established from 

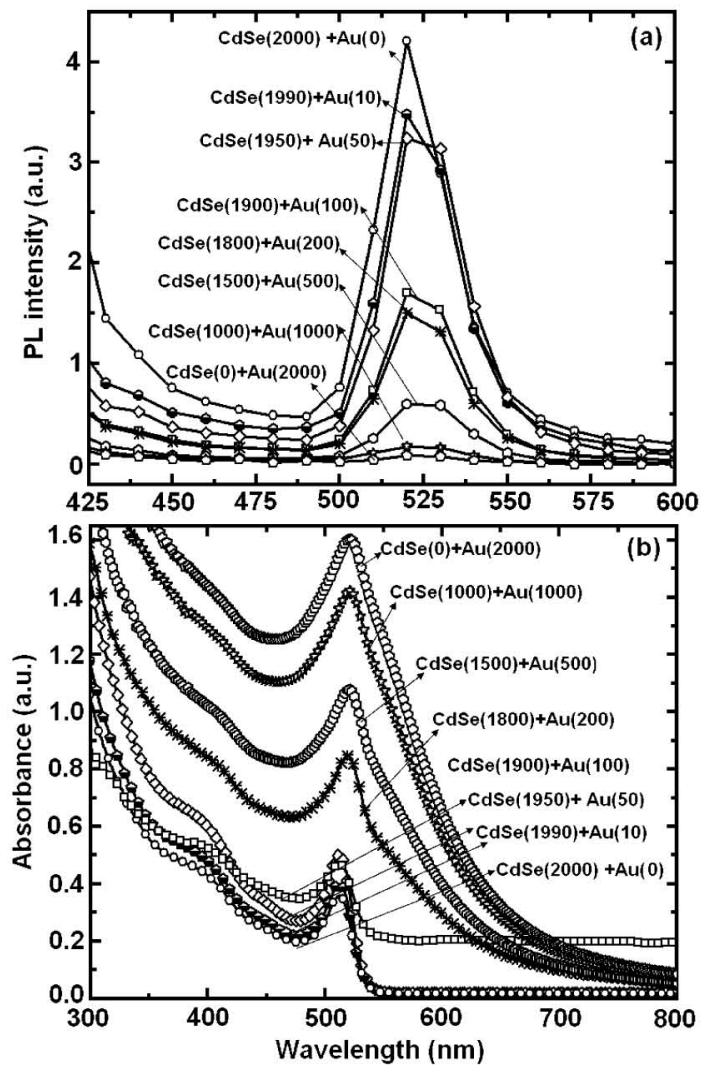

Figure 1. (a) PL spectra at an excitation wavelength of $405 \mathrm{~nm}$ and (b) absorption spectra in 300-1100 wavelength region of neat $(\mathrm{O}) \mathrm{CdSe}$ $(2000)+\mathrm{Au}(0)$, (half-filled pentagon) CdSe $(1990)+\mathrm{Au}(10),(\diamond)$ $\mathrm{CdSe}(1950)+\mathrm{Au}(50),(\square) \mathrm{CdSe}(1900)+\mathrm{Au}(100),(*) \mathrm{CdSe}(1800)$ $+\mathrm{Au}(200),(0) \mathrm{CdSe}(1500)+\mathrm{Au}(500)$, (전) CdSe $(1000)+\mathrm{Au}$ $(1000),(\Delta) \mathrm{CdSe}(0)+\mathrm{Au}(2000)$ colloidal solutions in toluene. The numbers in parentheses have the units of microliters. CdSe (initial concentration $)=20 \mathrm{mM}$ and $\mathrm{Au}($ solution strength $)=12.9 \mathrm{mM}$; both in toluene.

the literature ${ }^{29}$ that the quenching of the PL emission is the result of the electron transfer from semiconductor QDs to metal NPs. The PL quenching efficiency also depends on the separation distance between CdSe QDs and Au NPs. The decrease in the intensity of the emission peak of CdSe QDs continues until a certain concentration of the $\mathrm{Au}: \mathrm{CdSe}(1: 1$ by volume) is acquired. Thereafter, further addition of Au NPs to the CdSe solution manifests in a discontinuous variation in the emission. Hence, the concentration of Au NPs corresponding to maximum quenching approximates to the distance of the closest approach between Au NPs and CdSe QDs. At this fixed volume ratio of $\mathrm{Au}: \mathrm{CdSe}$, the electron-transfer rate from the conduction band of the CdSe QDs to the fermi level of the Au NPs ${ }^{29}$ is enhanced in comparison to other ratios and corresponds to maximum charge transfer. Consequently, this Au:CdSe solution composition was used for nanocomposite formation with PEDOT.

To add further credence to this 1:1 Au:CdSe colloid composition, absorption spectra of CdSe QDs at different loadings of Au NPs were also recorded (Figure 1b). Neat CdSe QDs in toluene show an absorption peak at $522 \mathrm{~nm}$, and this absorption peak due to surface-plasmon oscillations intensifies with increasing $\mathrm{Au}$ NPs, and at Au:CdSe (1:1) volume ratio, $\lambda_{\max }$ displays the highest optical density. The $\lambda_{\max }$ of the Au:CdSe is close to the position of $\pi-\pi^{*}$ absorption of neutral PEDOT $\left(\lambda_{\max } \approx 590 \mathrm{~nm}\right),{ }^{30}$ which indicates the suitability of $\mathrm{Au}-\mathrm{CdSe}$ for forming a nanocomposite with PEDOT.
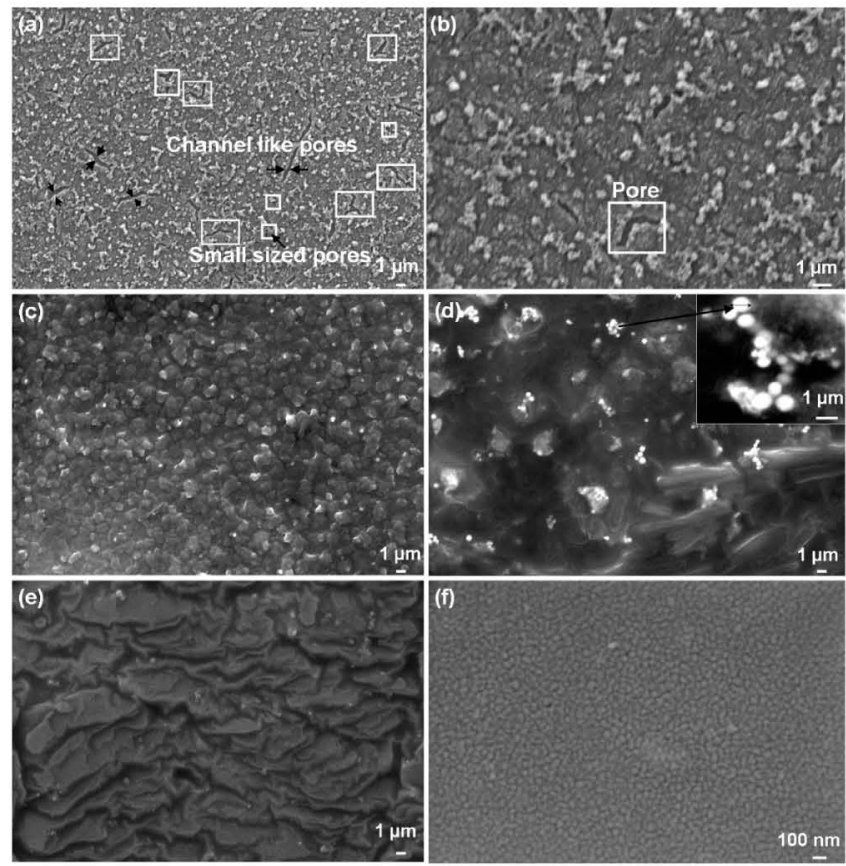

Figure 2. SEM micrographs of neat PEDOT (IL) at (a) low and (b) high magnifications, (c) PEDOT (IL)-CdSe, (d) PEDOT (IL)-Au with inset corresponding to the high-magnification image of bright white entities which are Au nanoclusters, (e) low- and (f) high-magnification images of PEDOT (IL)-Au-CdSe nanocomposite films.

3.2. Microstructural Studies of the Nanocomposite Film. The surface morphology of PEDOT (IL), PEDOT (IL)-CdSe, PEDOT (IL)-Au, and PEDOT (IL)-Au-CdSe are shown in the SEM micrographs (Figure 2). Neat PEDOT (IL) film (Figure 2a) shows a uniform granular surface morphology wherein small-sized randomly distributed pores with diameters varying from 100 to $200 \mathrm{~nm}$ are seen. The pores in some portions have channel-like shapes with a width as large as $500 \mathrm{~nm}$, and these are clearly perceptible in the high-magnification image in Figure 2b. The SEM image of PEDOT (IL)-CdSe (Figure 2c) composite shows an altered structure. Large agglomerates with diameters varying between $700 \mathrm{~nm}$ and $2 \mu \mathrm{m}$ are seen to be distributed on the surface of the film (Figure 2c). The surface morphology of the PEDOT (IL)-Au composite (Figure $2 \mathrm{~d}$ and inset) is strikingly different; the bright white spots in the micrograph are the Au NP clusters, and their diameters vary from $500 \mathrm{~nm}$ to $1 \mu \mathrm{m}$. The morphology of the PEDOT (IL)-Au-CdSe film (Figure 2e) in certain portions shows foldings, which are due to the adsorption of wet $\mathrm{Au}-\mathrm{CdSe}$ colloids on the surface by solution-cast method. The granular texture of the film is visible in the high-magnification image of the PEDOT (IL)- $\mathrm{Au}-\mathrm{CdSe}$ film as shown in Figure 2f.

Au NPs and CdSe QDs can be distinguished from one another in the HRTEM micrographs shown in Figure 3a,b. The Au NP diameter is about $4-5 \mathrm{~nm}$ (Figure $3 \mathrm{a}$ ), and the CdSe QD size ranges between 3 and $4 \mathrm{~nm}$ (Figure 3b). In general, singlecrystalline Au NPs are oriented along the 111 and 200 planes with interplanar spacings of 0.24 and $0.22 \mathrm{~nm}$, respectively. Similarly, CdSe QDs are oriented along the 111 and 220 reflections with interplanar spacings of 0.35 and $0.21 \mathrm{~nm}$, respectively. Insets in Figure 3a,b on left-hand side show interplanar spacings of 0.24 and $0.35 \mathrm{~nm}$, for $\mathrm{Au} \mathrm{NP}$ and CdSe QD, respectively. Fast Fourier transformation (FFT) on latticescale images of Au NP and CdSe QD (insets in Figure 3a and $\mathrm{b}$ on right-hand side) clearly reveal spotty electron-diffraction patterns with the outermost ring in both cases attributable to 


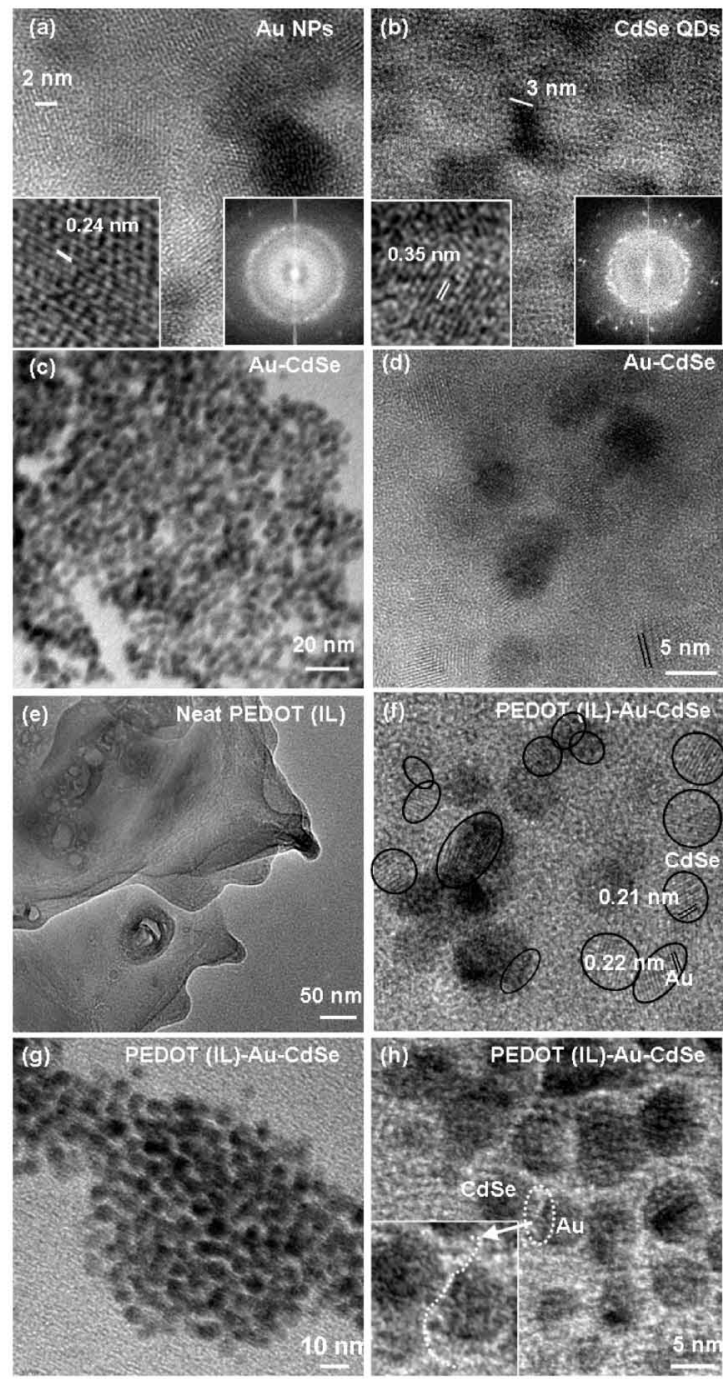

Figure 3. HRTEM micrographs of (a) Au NPs, (b) CdSe QDs, (c) and (d) $\mathrm{Au}-\mathrm{CdSe}$, (e) neat PEDOT (IL), and (f-h) PEDOT (IL)$\mathrm{Au}-\mathrm{CdSe}$ nanocomposite films; the ellipsoids in panel $\mathrm{f}$ ensconce the lattice planes of $\mathrm{Au} / \mathrm{CdSe}$. The black lines in panel f show orientations along two different planes. Insets on right hand side in panels $a$ and $b$ are the FFT performed on lattice-scale images of Au NPs and CdSe QDs, wherein the outermost ring of white spots corresponds to the (111) plane. Insets on the left hand side of panels a and b show the corresponding inter-planar spacings. Inset of panel $h$ is a magnified view of the interface of an Au NP and a CdSe QD.

the 111 plane. HRTEM images of Au-NPs-CdSe-QDs nanocomposite are shown in Figure 3c,d. The neat PEDOT (IL) film is characterized by a featureless micrograph, typical of the amorphous polymer (Figure 3e). The micrographs of PEDOT (IL)- $\mathrm{Au}-\mathrm{CdSe}$ nanocomposite are shown in Figure $3 \mathrm{f}-\mathrm{h}$. We have elucidated several such ultrafine nanocrystallites of $\mathrm{Au}$ and $\mathrm{CdSe}$ in Figure 3f. Inset in Figure 3h shows a clean interface between these NPs. At higher magnifications, we have noted that these NPs (Figure 3f,g) belong to Au (0.22 nm, plane 200) and CdSe (0.21 nm, plane 220). Because Au and CdSe are both cubic, a good interfacing between them is crystallographically agreeable. Figure $3 \mathrm{f}$ shows CdSe QDs and Au NPs with the interplanar spacings of 0.22 and $0.21 \mathrm{~nm}$, respectively. However, because the interplanar spacings of 200 planes of $\mathrm{Au}(0.22 \mathrm{~nm})$ and 220 planes of CdSe $(0.22 \mathrm{~nm})$ are not very different, there will be a mismatch at the interface between the two. The interconnectivity between Au NPs and CdSe QDs provides evidence for charge transfer at the lattice scale.
3.3. XPS Studies of the Nanocomposite Film. The formation of the PEDOT (IL)-Au-CdSe nanocomposite was confirmed from elemental composition deduced from the XPS corelevel spectra of $\mathrm{Au}, \mathrm{Cd}$, and Se shown in Figure 4. After fixing the fwhm at $0.68 \mathrm{eV}$, the Au photoemission was deconvoluted into a doublet centered at 83.4 and $87.2 \mathrm{eV}$ (Figure 4a) attributable to the $\mathrm{Au} 4 \mathrm{f}_{7 / 2}$ and $\mathrm{Au} 4 \mathrm{f}_{5 / 2}$ respectively. ${ }^{28}$ Because the positions are slightly upshifted in comparison to metallic $\mathrm{Au},{ }^{28}$ it is an indirect evidence of further charge transfer from $\mathrm{Au}$ to PEDOT. The absence of a band at $84.9 \mathrm{eV}$, which is assigned to $\mathrm{Au}^{+},{ }^{28,31}$ indicates that the $\mathrm{Au}$ atoms are not in the oxidized state in the nanocomposite. For $\mathrm{Cd} 3 \mathrm{~d}$ ( $\mathrm{fwhm} \approx 1.75$ $\mathrm{eV}$, Figure $4 \mathrm{~b}$ ), peaks at 404.0 and $410.4 \mathrm{eV}$ arise from the doublet of $\mathrm{Cd} 3 \mathrm{~d}_{5 / 2}$ and $\mathrm{Cd} 3 \mathrm{~d}_{3 / 2}$, respectively. ${ }^{32}$ Appearance of $\mathrm{Cd} 3 \mathrm{~d}_{5 / 2}$ indicates that $\mathrm{Cd}$ prevails in +2 oxidation state, ${ }^{33}$ thus confirming complete charge transfer from the QDs to the metallic NPs. The deconvolution of the Se $3 \mathrm{~d}$ (fwhm $\approx 2.9$ $\mathrm{eV}$, Figure $4 \mathrm{c}$ ) resulted in three peaks characteristic of the three oxidation states. The peaks at 52.1, 57.9, and $60.7 \mathrm{eV}$ indicate $\mathrm{Se}^{2-}$ (reduced), $\mathrm{Se}^{0}$ (neutral), and $\mathrm{Se}^{2+}$ (oxidized) states of $\mathrm{Se}$. Charge transfer from $\mathrm{CdSe}$ to $\mathrm{Au}$ is responsible for the appearance of $\mathrm{Se}^{0}$ and $\mathrm{Se}^{2+}$ signals. Generally, the chemical shift between $\mathrm{Se}^{0}$ and $\mathrm{Se}^{2-}$ lies in the range of $1-2 \mathrm{eV} ;{ }^{34,35}$ but in the present case, it is larger than the reported values, because the reported values are based on deconvoluted spectra, whereas here, we obtained two well-resolved peaks experimentally. In a similar way, $\mathrm{Lu}$ et. al also obtained an Se3d signal spanning from $\sim 51$ to $60 \mathrm{eV}$, with two independent contributions, and the peak separation between reduced Se and elemental Se was about $3 \mathrm{eV} \cdot{ }^{36}$ A literature survey on Se $3 \mathrm{~d}$ shows that the corelevel spectrum tends to extend over a rather broad binding energy range of $50-62 \mathrm{eV},{ }^{34-38}$ indicating that Se can produce signals within this range, depending on its oxidation state and chemical environment.

In order to further confirm that the Au-NPs-CdSe-QDs are distributed across the cross section of the film, the PEDOT (IL)$\mathrm{Au}-\mathrm{CdSe}$ nanocomposite film was also subjected to sputtering, and the XPS spectrum before and after sputtering is shown in Figure $5 \mathrm{a}, \mathrm{b}$. The presence of distinctive signals from $\mathrm{Au}, \mathrm{Cd}$, and $\mathrm{Se}$ in the sputtered specimen confirmed that $\mathrm{Au}$ and $\mathrm{CdSe}$ nanostructures are homogeneously distributed in the composite; indeed, if they had been merely confined to the surface of the film, the sputtered sample would not have been characterized by contributions from these nanomoieties. The sulfur core-level spectra of PEDOT (IL) (Figure 5c) and PEDOT (IL)-Au-CdSe (Figure 5d) nanocomposite film are clearly distinguishable. In concurrence with literature reports ${ }^{39,40}$ herein, each contribution in the $\mathrm{S} 2 \mathrm{p}$ core-level spectra exhibits a spin-split doublet due to $S 2 p_{3 / 2}$ and $S 2 p_{1 / 2}$ with an energy difference of $\sim 1.2 \mathrm{eV}$ and an intensity ratio of $2: 1$. In PEDOT (IL), when resolving the main peak, the $S 2 p_{3 / 2}$ and $S 2 p_{1 / 2}$ peaks of PEDOT appear at 164.0 and $165.2 \mathrm{eV}$, respectively, whereas the sulfur components in $\mathrm{N}\left(\mathrm{CF}_{3} \mathrm{SO}_{2}\right)_{2}{ }^{-}$(of ionic liquid counterion) appear at 168.6 and $169.8 \mathrm{eV}$. In PEDOT (IL)-Au-CdSe nanocomposite film (Figure 5d), an additional set of peaks corresponding to the $\mathrm{S}-\mathrm{Au}$ interaction are observed at 165.7 and $166.9 \mathrm{eV}$, which confirms the linkage of Au NPs with the sulfur atoms on the thiophene rings in PEDOT.

The density of states close to the fermi level corresponding to zero are shown in Figure 4d. The estimated energy difference between the valence band and fermi level is $1.1 \mathrm{eV}$ in the PEDOT (IL)-Au-CdSe nanocomposite, whereas in neat PEDOT (IL) film, it is about $1.43 \mathrm{eV}$, indicative of a lower-band 


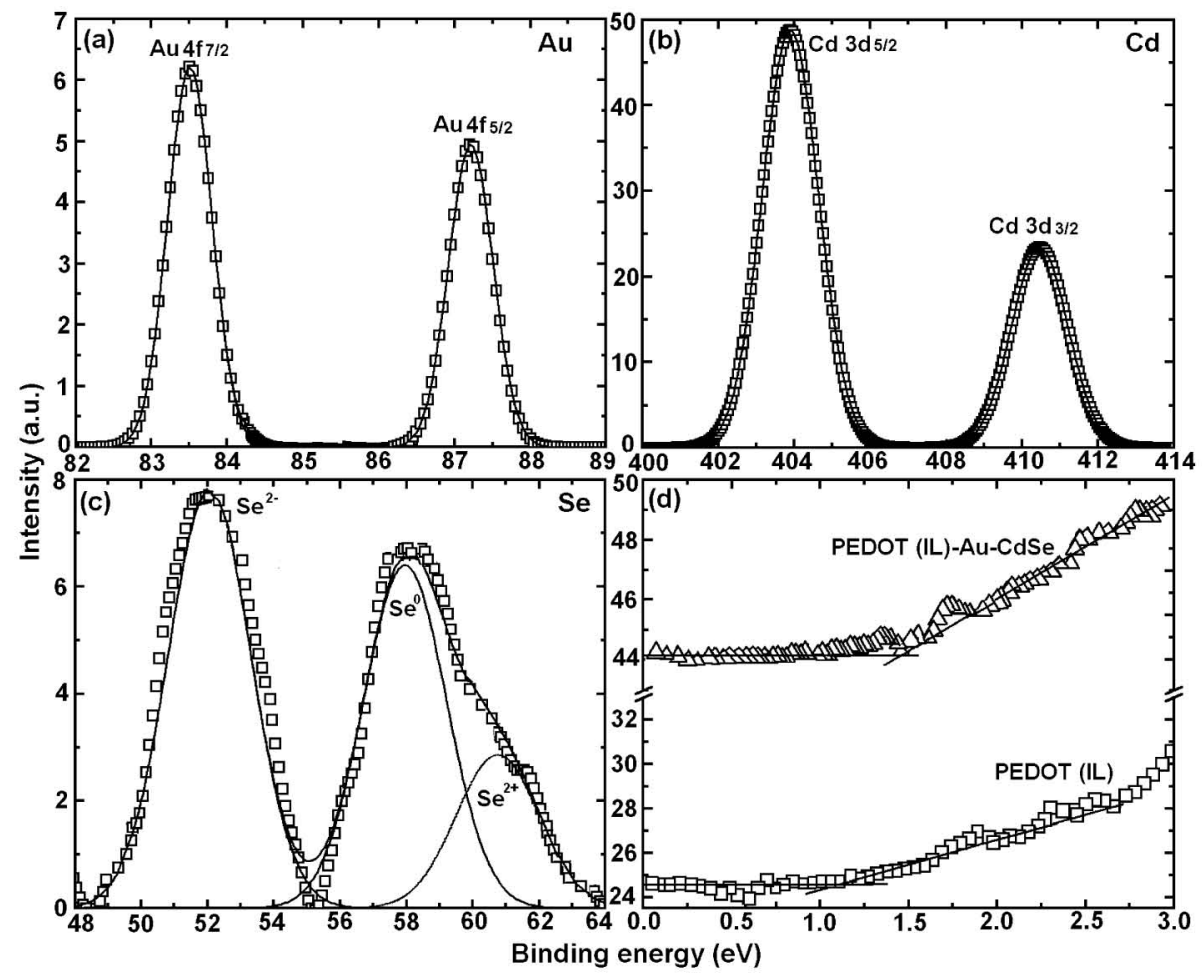

Figure 4. Core level spectra of PEDOT (IL)-Au-CdSe nanocomposite film with solid lines signifying the deconvoluted contributions of (a) Au 4f, (b) Cd 3d, and (c) Se 3d, and (d) valence-band spectra of neat PEDOT (IL) ( $\square$ ) and PEDOT (IL)-Au-CdSe nanocomposite ( $\triangle$ ) displaying the respective Fermi edges.

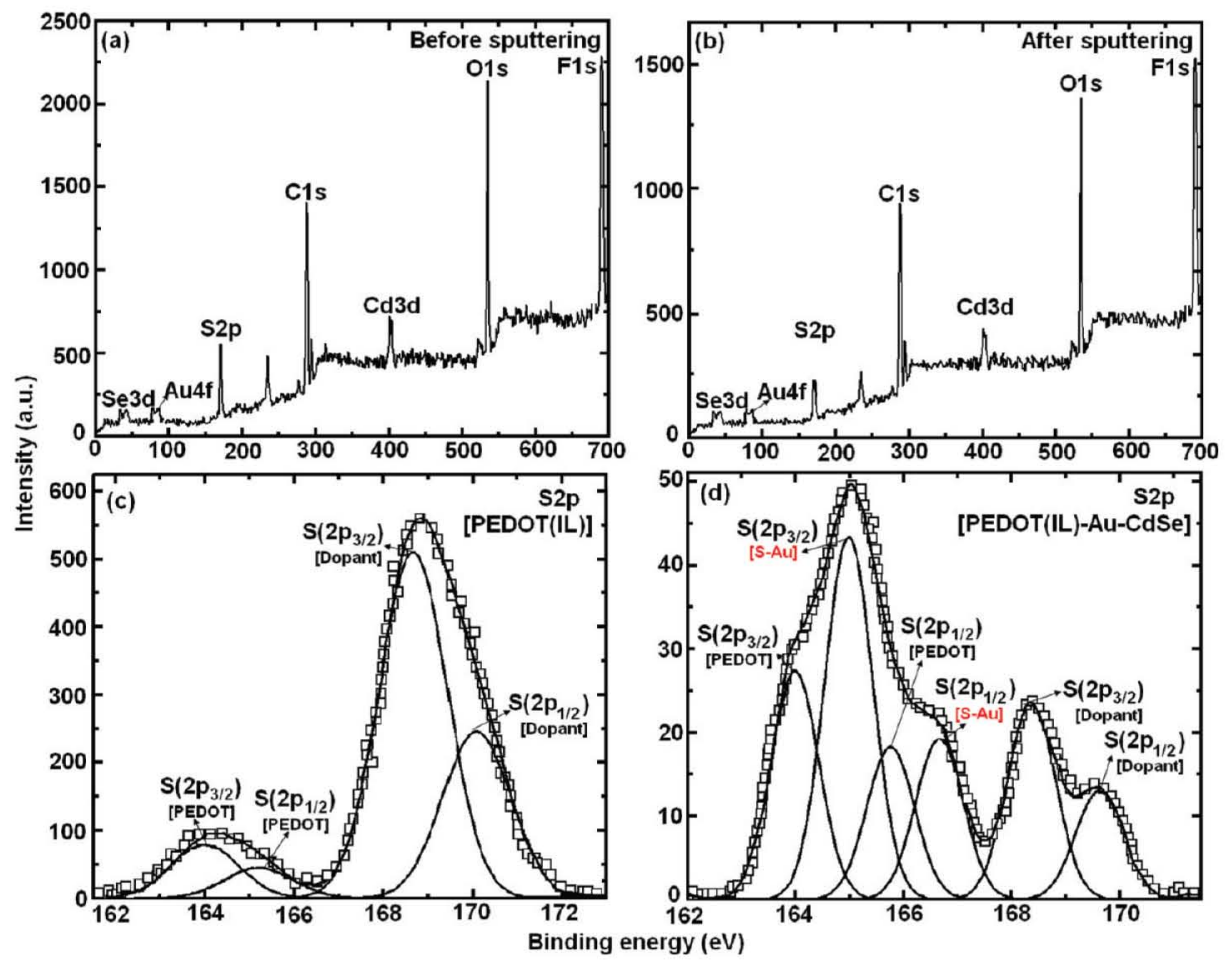

Figure 5. XPS survey spectrum of a PEDOT (IL)-Au-CdSe nanocomposite film (a) before and (b) after sputtering and deconvoluted S $2 p$ corelevel spectra of (c) neat PEDOT (IL) and (d) PEDOT (IL)-Au-CdSe nanocomposite films.

gap in the former. The implications are described in detail in the next section.

3.4. Optical Studies of the Nanocomposite Film. Chargetransfer phenomena in the PEDOT (IL)-Au-CdSe nanocomposite film was followed by PL studies (Figure 6a). The PL emission peak experiences a significant intensity enhancement ongoing from neat PEDOT (IL) film to the PEDOT (IL)$\mathrm{Au}-\mathrm{CdSe}$ nanocomposite film, indicating that electrons are transported from $\mathrm{Au}-\mathrm{CdSe}$ to PEDOT in the nanocomposite film. This emission peak also shows a bathochromic shift as it moves from 405 to $416 \mathrm{~nm}$ ongoing from neat PEDOT (IL) film to the PEDOT (IL)-Au-CdSe composite film. PL spectra of the PEDOT (IL)-CdSe and PEDOT (IL)-Au films were also examined. The PL peak intensities of neat PEDOT (IL) and PEDOT (IL)-Au are comparable, indicating that pristine Au NPs have no measurable effect on PEDOT (IL). Of the two films, 


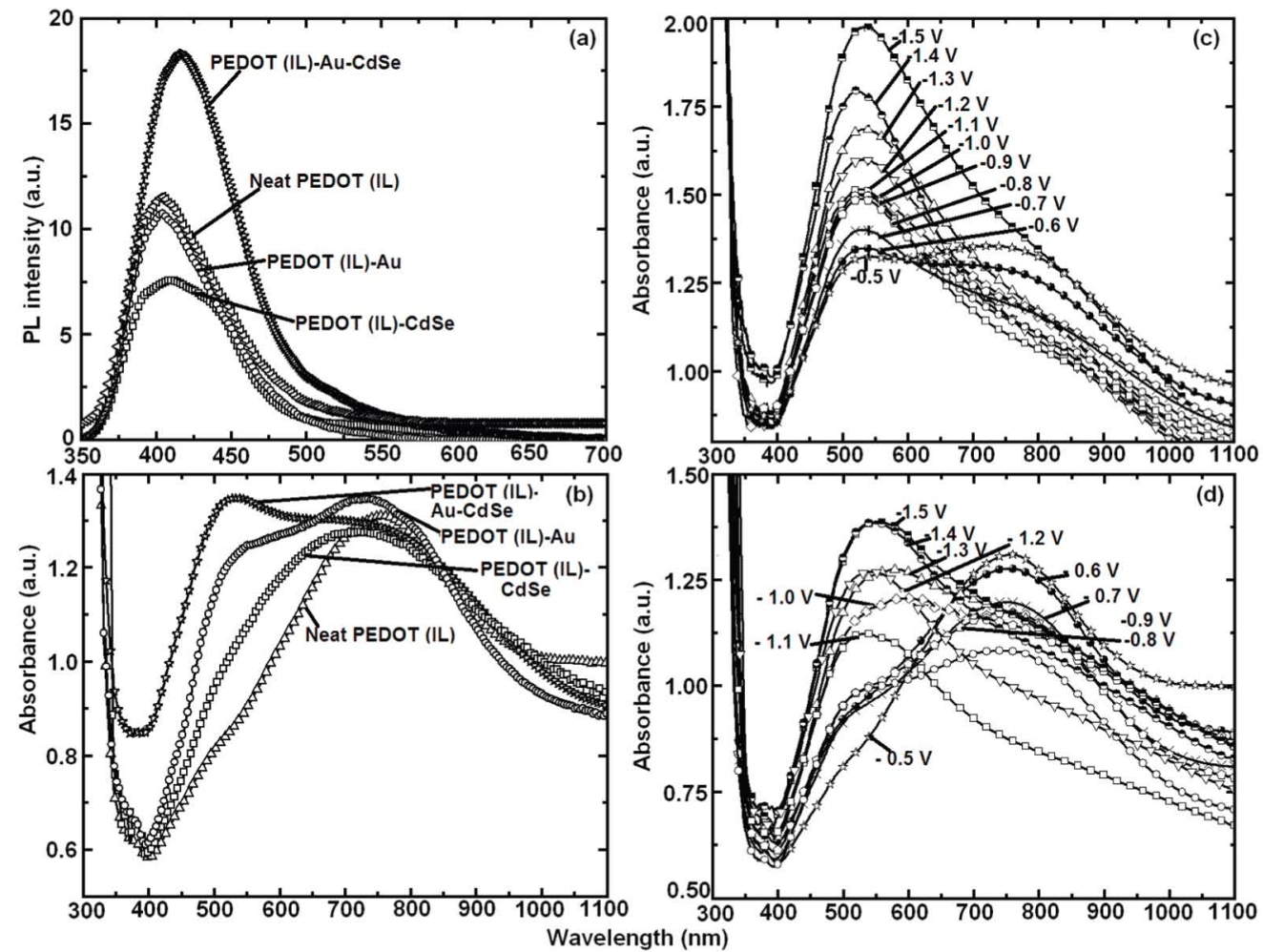

Figure 6. (a) Photoluminescence spectra recorded at an excitation wavelength of $405 \mathrm{~nm}$ and (b) absorption spectra in $300-1100$ wavelength region of (ङ) PEDOT (IL)-Au-CdSe nanocomposite, ( $\nabla$ ) neat PEDOT (IL), (O) PEDOT (IL)-Au and ( $\square$ ) PEDOT (IL)-CdSe films, absorption spectra of (c) PEDOT (IL)-Au-CdSe nanocomposite and (d) neat PEDOT (IL) film recorded under different reduction potentials: -0.5 ( $(\bullet),-0.7(\times),-0.8(\Delta),-0.9(\bigcirc),-1.0(\diamond),-1.1(\square),-1.2(\nabla),-1.3(\Delta),-1.4(\vartheta)$ and $-1.5(\Xi) \mathrm{V}$ in steps of $-0.1 \mathrm{~V}$.

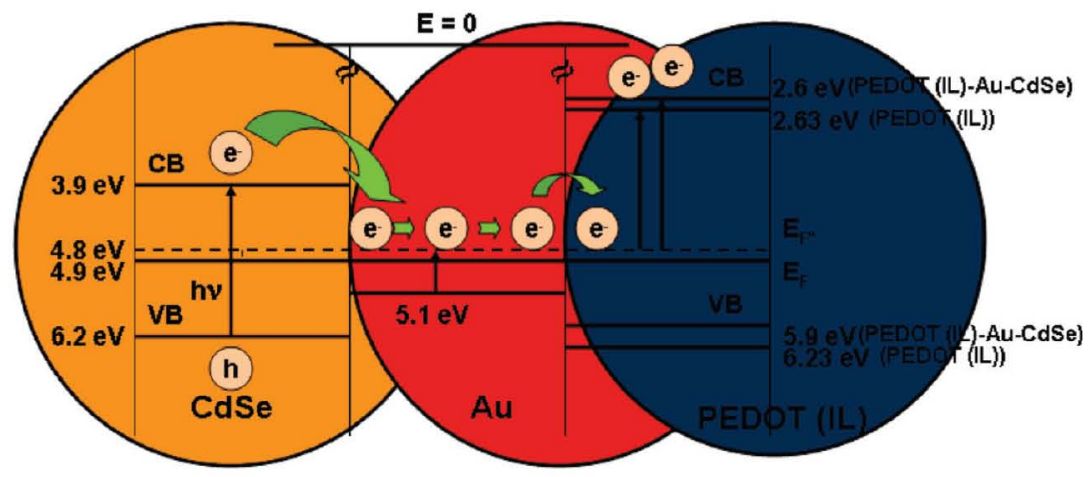

Figure 7. Schematic showing the Fermi-level equilibration in PEDOT (IL)-Au-CdSe nanocomposite system.

the PEDOT (IL)-CdSe film has the lowest emission intensity (lower than neat PEDOT (IL)), which shows that electron transfer occurs from the polymer to the CdSe QDs ${ }^{23}$ Hence, it is inferred that the optical response of PEDOT (IL)- $\mathrm{Au}-\mathrm{CdSe}$ is unique and cannot be obtained by using either Au NPs or CdSe QDs independently with the polymer. The charge-transfer mechanism in the PEDOT (IL)-Au-CdSe nanocomposite has been described on the basis of Fermi-level equilibration ${ }^{41}$ in Figure 7. The neat PEDOT (IL) shows the $764 \mathrm{~nm}$ peak corresponding to bipolaronic states, corresponding to the oxidized state, whereas this peak is suppressed in the PEDOT (IL)-Au-CdSe nanocomposite indicating that PEDOT is not fully oxidized.

On introducing the Au NPs to the CdSe QD solution, the photogenerated electrons migrate from the conduction band of the semiconducting CdSe QDs to the metallic Au NPs. The overall Fermi level shifts to more negative potentials, that is, from 4.9 to $4.8 \mathrm{eV}$, eventually equilibrating with that of the CdSe QDs. When the $\mathrm{Au}-\mathrm{CdSe}$ system is brought in direct contact with PEDOT, because of the extension of Fermi-level equilibration, electrons migrate from electron-rich Au to the Fermi level of the polymer. The negative shift in the fermi level of the nanocomposite implies a higher degree of electron accumulation which is reflected in the absorption spectrum of the PEDOT (IL)-Au-CdSe nanocomposite in Figure $6 \mathrm{~b}$. However, this effect cannot be realized in neat PEDOT (IL) film where no electron-donating moiety prevails. The distance of the Fermi level from the valence-band and band-gap values deduced from the XPS valence-band spectra as well as absorbance spectra were utilized to deduce the positions of the conduction band of the PEDOT (IL) and PEDOT (IL)-Au-CdSe composite films.

The PEDOT (IL)-CdSe film shows a broad absorption in the $740-840 \mathrm{~nm}$ wavelength range spanning over $100 \mathrm{~nm}$, and the PEDOT (IL)-Au film shows the surface plasmonic peak at 541 $\mathrm{nm}$ and an oxidation peak at $728 \mathrm{~nm}$ indicating that PEDOT (IL)-Au is analogous to PEDOT (IL) in terms of the polymer contribution. In the PEDOT (IL)-Au-CdSe nanocomposite film, 

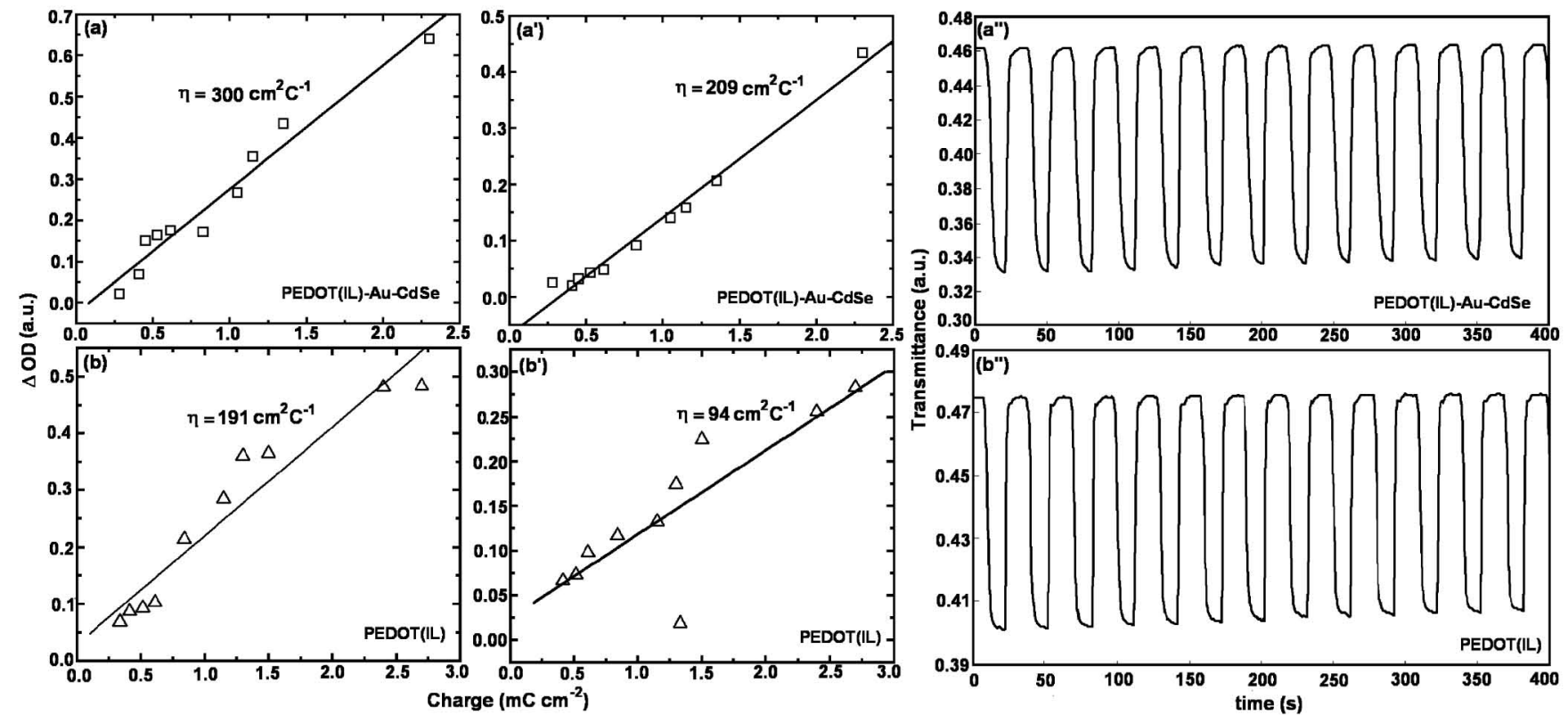

Figure 8. Optical density change in ( $\square$ ) PEDOT (IL)-Au-CdSe and ( $\triangle$ ) neat PEDOT (IL) films as a function of intercalated charge density at 550 (a and b) and 633 ( $a^{\prime}$ and b') nm, respectively, with optical density at $-0.5 \mathrm{~V}$ taken as reference. ( $\left.\mathrm{a}^{\prime \prime}\right)$ and $\left(\mathrm{b}^{\prime \prime}\right)$ show the transmittance versus time transients of PEDOT (IL)-Au-CdSe and neat PEDOT (IL) films at a monochromatic wavelength of $632.8 \mathrm{~nm}$ under a square-wave dc potential of $\pm 1.5 \mathrm{~V}$.

the enhanced intensity of the $500 \mathrm{~nm}$ absorption indicates a contribution from $\pi-\pi^{*}$ of PEDOT, again corroborating with the increased reduction level of PEDOT. Spectroelectrochemistry of the PEDOT (IL)-Au-CdSe nanocomposite and neat PEDOT (IL) films were studied in the ionic liquid 1-butyl-1methylpyrrolidinium bis(trifluoromethylsulfonyl)imide, by increasing reduction potential from -0.5 to $-1.5 \mathrm{~V}$ in steps of $0.1 \mathrm{~V}$ (Figure $6 \mathrm{c}, \mathrm{d}$ ). For the neat PEDOT (IL) film (Figure 6d), the bipolaronic transition peak is prominent in the range from -0.5 to $-0.9 \mathrm{~V}$, whereas the same peaks have lower intensities in the nanocomposite film (Figure 6c). For both films, with increase in the reduction potential, the $\pi-\pi^{*}$ transition peak gains intensity at the expense of the bipolaronic peak. This $\pi-\pi^{*}$ transition peak appears in neat PEDOT (IL) film at -0.8 $\mathrm{V}$, whereas in the PEDOT (IL)-Au-CdSe nanocomposite film, it is perceptible even at $-0.5 \mathrm{~V}$, which shows that a lower ion loading suffices to induce a larger change in optical density in the PEDOT (IL)-Au-CdSe film in comparison to the neat PEDOT (IL) film.

The coloration efficiency or change in optical density $(\triangle \mathrm{OD})$ as a function of charge ingress/egress of PEDOT (IL)-AuCdSe nanocomposite and neat PEDOT (IL) films are shown in Figure $8 \mathrm{a}, \mathrm{b}$ at $550 \mathrm{~nm}$ and in Figure $8 \mathrm{a}^{\prime}, \mathrm{b}$ ' at $633 \mathrm{~nm}$. The reference state was set at $-0.5 \mathrm{~V}$, corresponding to injected charge densities of 0.18 and $0.24 \mathrm{mC} \mathrm{cm}^{-2}$ for PEDOT (IL)$\mathrm{Au}-\mathrm{CdSe}$ nanocomposite and PEDOT (IL) films, respectively. The reason for choosing this reference state is provided in the Supporting Information. The coloration efficiency of the PEDOT (IL)-Au-CdSe $(\lambda=550 \mathrm{~nm})$ is $300 \mathrm{~cm}^{2} \mathrm{C}^{-1}$ (Figure 8a), whereas in neat PEDOT (IL), it is only $191 \mathrm{~cm}^{2} \mathrm{C}^{-1}$ (Figure $8 b$ ). The coloration efficiency of the nanocomposite film is greater than thepreviously reported values of $183(\lambda=585 \mathrm{~nm})$ by Reynolds et al., ${ }^{42} 181(\lambda=655 \mathrm{~nm})$, and $206 \mathrm{~cm}^{2} \mathrm{C}^{-1}(\lambda=$ $570 \mathrm{~nm}$ ) by Ho et al. ${ }^{43,30}$ for PEDOT. The calculated coloration efficiency is comparable with the well-known analogues of PEDOT, that is poly (3,4-propylenedioxythiophene) (PProDOT) of $285 \mathrm{~cm}^{2} \mathrm{C}^{-1}(\lambda=578 \mathrm{~nm})$ and also to that of its derivative poly-(3,3-dimethyl-3,4-dihydro-2H-thieno[3,4- $b$ ]dioxepine) (PProDOT- $\left.\mathrm{Me}_{2}\right)\left(375 \mathrm{~cm}^{2} \mathrm{C}^{-1}, \lambda=585 \mathrm{~nm}\right)$, reported by Gaupp et al. ${ }^{42}$ At $633 \mathrm{~nm}$, the coloration efficiency decreases to $209 \mathrm{~cm}^{2}$ $\mathrm{C}^{-1}$ (Figure 8a') for PEDOT (IL)-Au-CdSe, and for neat PEDOT (IL), it is considerably low, that is, $94 \mathrm{~cm}^{2} \mathrm{C}^{-1}$ (Figure $\left.8 b^{\prime}\right)$, reaffirming the role of $\mathrm{Au}-\mathrm{CdSe}$ in improving the electrochromic response of the PEDOT.

Optical-switching studies of the two films performed under a square-wave potential of $\pm 1.5 \mathrm{~V}$ at $632.8 \mathrm{~nm}$ at $0.03 \mathrm{~Hz}$ are shown in Figure $8 a^{\prime \prime}$ and $b^{\prime \prime}$. The transmission modulation of the neat PEDOT (IL) (Figure $8 b^{\prime \prime}$ ) is only $60 \%$ of the that of the PEDOT (IL)-Au-CdSe nanocomposite film (Figure 8a") owing to the ability of the nanocomposite to incur a higher optical change for the same value of applied potential. To enable a comparison of the two systems, coloration and bleaching times of the two films of comparable areas and thicknesses were determined by considering the time taken to bring about a $90 \%$ transmission change of the optical contrast. The neat PEDOT (IL) film requires $5.2 \mathrm{~s}$ to color and $3.1 \mathrm{~s}$ to bleach, whereas the PEDOT (IL)- $\mathrm{Au}-\mathrm{CdSe}$ nanocomposite film shows a faster kinetic response; it colors in $4.5 \mathrm{~s}$ and bleaches in just $1.5 \mathrm{~s}$, indicating that nanocomposite films are most conducive for electrochromic applications.

\section{Conclusions}

PEDOT-Au-CdSe nanocomposite films were synthesized by sequential electropolymerization first of PEDOT, in a hydrophobic ionic liquid 1-butyl-1-methylpyrrolidinium bis(trifluoromethylsulfonyl)imide, followed by immersion in a Au:CdSe (1:1, by volume) colloid to yield a film, with a homogeneous distribution of the Au: $\mathrm{CdSe}$ nano clusters across the cross section of the conjugated polymer film. Increment in the surface-plasmon absorption of $\mathrm{Au}$ and decrease in the PL emission $\lambda_{\max }$ corresponding to $\mathrm{CdSe}$, in the photopic region achieved as a function of Au-NP addition to CdSe-QD solution, ratified that charge transfer occurs from CdSe to $\mathrm{Au}$. Further evidence for charge transport in the film from CdSe to $\mathrm{Au}$ to PEDOT was obtained in the form of signals corresponding to oxidized Se in XPS spectra and PL quenching and absorption data. The influence of Au:CdSe nanostructures was reflected in the remarkably high coloring efficiency, large absorption change, 
faster blue-to-pale-blue reversible switching rates as compared to the performance of the control sample. We demonstrated for the first time that CdSe QDs, when utilized in conjunction with $\mathrm{Au}$ NPs, play a pivotal role in controlling and modifying the electrochromism and electroactivity of a conducting polymer like PEDOT.

\section{Synopsis}

The surface-plasmon absorption of Au NPs and the ability of CdSe QDs to act as electron conduits, when synergistically combined with the high electrochromic contrast of PEDOT, yield a PEDOT-Au-CdSe nanocomposite film which shows a remarkably high optical density change and superior electrochemical activity when compared with the performance of a film devoid of the $\mathrm{Au}-\mathrm{CdSe}$ nanostructures.

Acknowledgment. S.B. acknowledges University Grants Commission (UGC) for senior research fellowship. We thank Dr. S.T. Lakshmikumar, National Physical laboratory for his guidance and encouragement. Financial support from DST (DST/TSG/PT/2007/69) is gratefully acknowledged.

Supporting Information Available: Absorbance plots of PEDOT (IL) film and PEDOT(IL)-Au-CdSe nanocomposite film under different potentials in the ionic liquid 1-butyl-1methylpyrrolidinium bis(trifluoromethylsulfonyl)imide. This material is available free of charge via the Internet at http:// pubs.acs.org.

\section{References and Notes}

(1) Rao, C. N. R.; Cheetham, A. K. Science and Technology of Nanomaterials: Current Status and Future Prospects. J. Mater. Chem. 2001, $11,2887-2894$.

(2) Gomez-Romero, P. Hybrid Organic-Inorganic Materials - In Search of Synergic Activity. Adv. Mater. 2001, 13, 163-174.

(3) Henglein, A. Radiolytic Preparation of Ultrafine Colloidal Gold Particles in Aqueous Solution: Optical Spectrum, Controlled Growth and Some Chemical Reactions. Langmuir 1999, 15, 6738-6744.

(4) Tan, Y.; Li, Y.; Zhu, D. Synthesis of Poly(2-methoxyaniline)/Au Nanoparticles in Aqueous Solution with Chlorauric Acid as the Oxidant. Synth. Met. 2003, 135-136, 847-848.

(5) Athawale, A. A.; Bhagwat, S. V. Synthesis and Characterization of Novel Copper/Polyaniline Nanocomposite and Application as a Catalyst in the Wacker Oxidation Reaction. J. Appl. Polym. Sci. 2003, 89, 2412-2417.

(6) Dai, X.; Tan, Y.; Xu, J. Formation of Gold Nanoparticles in the Presence of o-Anisidine and the Dependence of the Structure of Poly(oanisidine) on Synthetic Conditions. Langmuir 2002, 18, 9010-9016.

(7) Wang, S.; Li, C.; Shi, G. Photoresponsive Properties of Multilayers of Conductive Polymer and CdSe Nanoparticles. Sol. Energy Mater. Sol. Cells 2008, 92, 543-549.

(8) Caseri, W. Nanocomposites of Polymers and Metals or Semiconductors: Historical Background and Optical Properties. Macromol. Rapid Commun. 2000, 21, 705-722.

(9) Kamat, P. V. Photophysical, Photochemical and Photocatalytic Aspects of Metal Nanoparticles. J. Phys. Chem. B 2002, 106, 7729-7744.

(10) Sih, B. C.; Wolf, M. O. Metal Nanoparticle-Conjugated Polymer Nanocomposites. Chem. Commun. 2005, 3375-3384.

(11) Gangopadhyay, R.; De, A. Conducting Polymer Nanocomposites: A Brief Overview. Chem. Mater. 2000, 12, 608-622.

(12) Leroux, Y. R.; Lacroix, J. C.; Chane-Ching, K. I.; Fave, C.; Felidj, N.; Levi, G.; Aubard, J.; Krenn, J. R.; Hohenau, A. Conducting Polymer Electrochemical Switching as an Easy Means for Designing Active Plasmonic Devices. J. Am. Chem. Soc. 2005, 127, 16022-16023.

(13) Sarma, T. K.; Chattopadhyay, A. One Pot Synthesis of Nanoparticles of Aqueous Colloidal Polyaniline and Its Au-Nanoparticle Composite from Monomer Vapor. J. Phys. Chem. A 2004, 108, 7837-7842.

(14) Sarma, T. K.; Chowdhury, D.; Paul, A.; Chattopadhyay, A. Synthesis of Au Nanoparticle-Conductive Polyaniline Composite Using $\mathrm{H}_{2} \mathrm{O}_{2}$ as Oxidising as well as Reducing Agent. Chem. Commun. 2002, 1048-1049.

(15) Zhai, L.; McCullough, R. D. Regioregular Polythiophene/Gold Nanoparticle Hybrid Materials. J. Mater. Chem. 2004, 14, 141-143.

(16) Yen, B. K. H.; Stott, N. E.; Jensen, K. F.; Bawendi, M. G. A Continuous-Flow Microcapillary Reactor for the Preparation of a Size Series of CdSe Nanocrystals. Adv. Mater. 2003, 15, 1858.
(17) Qu, L.; Peng, X. Control of Photoluminescence Properties of CdSe Nanocrystals in Growth. J. Am. Chem. Soc. 2002, 124, 2049-2055.

(18) Han, M.; Gao, X.; Su, J. Z.; Nie, S. Quantum-dot-tagged Microbeads for Multiplexed Optical Coding of Biomolecules. Nat. Biotechnol. 2001, 19, 631-635.

(19) Choi, S.-H.; Song, H.; Park, I. K.; Yum, J.-H.; Kim, S.-S.; Lee, S.; Sung, Y.-E. J. Photochem. Photobiol. A 2006, 179, 135.

(20) Sofos, M.; Goldberger, J.; Stone, D. A.; Allen, J. E.; Ma, Q.; Herman, D. J.; Lauhon, W. L. J.; Stupp, S. I. A Synergistic Assembly of Nanoscale Lamellar Photoconductor Hybrids. Nat. Mater. 2009, 8, 68-75.

(21) Lu, G.; Li, C.; Shen, J.; Cheng, Z.; Shi, G. Preparation of Highly Conductive Gold-Poly(3,4-ethylenedioxythiophene) Nanocables and Their Conversion to Poly(3,4-ethylenedioxythiophene) Nanotubes. J. Phys. Chem. C 2007, 111, 5926-5931.

(22) Hsieh, S.-N.; Wen, T.-C.; Guo, T.-F. Polymer/Gold Nanoparticles Light Emitting Diodes Utilizing High Work Function Metal Cathodes. Mater. Chem. Phys. 2007, 101, 383-386.

(23) Sharma, H.; Sharma, S. N.; Singh, G.; Shivaprasad, S. M. Studies of Optical and Structural Properties of CdSe/Polymer Nanocomposites: Evidence of Charge Transfer and Photostability. Colloid Polym. Sci. 2007, $285,1213-1227$.

(24) Xu, J.; Hu, J.; Liu, X.; Qiu, X.; Wei, Z. Stepwise Self-Assembly of P3HT/CdSe Hybrid Nanowires with Enhanced Photoconductivity. Macromol. Rapid Commun. 2009, 30, 1419-1423.

(25) Oh, E.; Hong, M.-Y.; Lee, D.; Nam, S.-H.; Yoon, H. C.; Kim, H.S. Inhibition Assay of Biomolecules based on Fluorescence Resonance Energy Transfer (FRET) between Quantum Dots and Gold Nanoparticles. J. Am. Chem. Soc. 2005, 127, 3270-3271.

(26) Lu, W.; Wang, B.; Zeng, J.; Wang, X.; Zhang, S.; Hou, J. G Synthesis of Core/Shell Nanoparticles of Au/CdSe via Au-Cd Bialloy Precursor. Langmuir 2005, 21, 3684-3687.

(27) Sharma, S. N.; Pillai, Z. S.; Kamat, P. V. Photoinduced Charge Transfer between CdSe Quantum Dots and p-Phenylenediamine. J. Phys. Chem. B 2003, 107, 10088-10093.

(28) Brust, M.; Walker, M.; Bethell, D.; Schiffrin, D. J.; Whyman, R. Synthesis of Thiol-Derivatised Gold Nanoparticles in a Two-phase LiquidLiquid System. J. Chem. Soc., Chem. Commun. 1994, 801-802.

(29) Nikoobakht, B.; Burda, C.; Braun, M.; Hun, M.; El-Sayed, M. A. The Quenching of CdSe Quantum Dots Photoluminescence by Gold Nanoparticles in Solution. Photochem. Photobiol. 2002, 75, 591-597.

(30) Lin, T. H.; Ho, K. C. A Complementary Electrochromic Device Based on Polyaniline and Poly(3,4-ethylenedioxythiophene). Sol. Energy Mater. Sol. Cells 2006, 90, 506-520.

(31) Ertas, G.; Demirok, U. K.; Suzer, S. Enhanced Peak Separation in XPS with External Biasing. Appl. Surf. Sci. 2005, 249, 12-15.

(32) Nie, W.; He, J.; Zhao, N.; Ji, X. A Controllable Synthesis of MultiArmed CdTe Nanorods. Nanotechnolgy 2006, 17, 1146-1149.

(33) Chong, S. V.; Suresh, N.; Xia, J.; Al-Salim, N.; Idriss, H. TiO Nanobelts/CdSe Quantum Dots Nanocomposite. J. Phys. Chem. C 2007, 111, 10389-10393.

(34) Cachet, H.; Cortes, R.; Forment, M.; Etchberry, A. Electrodeposition of Epitaxial CdSe on (111) Gallium Arsenide. Thin Solid Films 2000, 316$362,84-87$.

(35) Calareso, C.; Grasso, V.; Silipigni, L. The Cadmium Seleniophosphate $\left(\mathrm{CdPSe}_{3}\right)$ XPS and XAES Spectra. Appl. Surf. Sci. 2002, 171, 306 313.

(36) Lu, W.; Sullivan, J. L.; Saied, S. O. XPS and LEISS Studies of Ion Bombarded GaSb, InSb and CdSe Surfaces. Surf. Sci. 1996, 352-354, 781-787.

(37) Feng, P. X.; Riley, J. D.; Leckey, R. C. G.; Pigram, P. J.; Hollering, M.; Ley, L. Surface and Bulk Properties of GaAs(001) Treated by Selenium Layers. Surf. Sci. 2000, 468, 109-121.

(38) Canava, B.; Vigneron, J.; Etcheberry, A.; Guillemoles, J. F.; Lincot, D. High Resolution XPS Studies of Se Chemistry of a $\mathrm{Cu}(\mathrm{In}, \mathrm{Ga}) \mathrm{Se}_{2}$ Surface. Appl. Surf. Sci. 2002, 202, 8-14.

(39) Li, C.; Imae, T. Electrochemical and Optical properties of Poly(3,4ethylenedioxythiophene) film Electropolymerized in an Aqueous Sodium Dodecyl Sulfate and Lithium Tetrafluroborate medium. Macromolecules 2004, 37, 2411-2416.

(40) Cho, S. I.; Choi, D. H.; Kim, S. H.; Lee, S. B. Electrochemical Synthesis and Fast Electrochromics of Poly(3,4-ethylenedioxythiophene) Nanotubes in Flexible Substrate. Chem. Mater. 2005, 17, 4564-4566.

(41) Jakob, M.; Levanon, H. Charge Distribution between UV-Irradiated $\mathrm{TiO}_{2}$ and Gold Nanoparticles: Determination of Shift in the Fermi Level. Nano Lett. 2003, 3, 353-358.

(42) Gaupp, C. L.; Welsh, D. M.; Rauh, R. D.; Reynolds, J. R. Composite Coloration Efficiency Measurements of Electrochromic Polymers Based on 3,4-Alkylenedioxythiophenes. Chem. Mater. 2002, 14, 3964-3970.

(43) Huang, S. W.; Ho, K. C. An All-Thiophene Electrochromic Device Fabricated with Poly(3-methylthiophene) and Poly(3,4-ethylenedioxythiophene). Sol. Energy Mater. Sol. Cells 2006, 90, 491-505. 